\title{
Critérios clínicos para diagnóstico e tratamento adequado da anafilaxia
}

\section{perioperatória}

\author{
Clinical criteria for proper diagnosis and treatment of perioperative anaphylaxis \\ Criterios clínicos para el diagnóstico y tratamiento adecuados de la anafilaxia perioperatória
}

Recebido: 05/10/2021 | Revisado: 12/10/2021 | Aceito: 14/10/2021 | Publicado: 16/10/2021

Luiz Fellipe Teixeira de Sousa ORCID: https://orcid.org/0000-0002-9476-6511 Instituto Tocantinense Antônio Carlos SA, Brasil

E-mail: fellipe.tsousa@gmail.com

Eduardo Augusto Souza Alves ORCID: https://orcid.org/0000-0003-3315-6928 Instituto Tocantinense Antônio Carlos SA, Brasil

E-mail: Eduaugusto89@icloud.com

Victor Matheus Gomes de Oliveira ORCID: https://orcid.org/0000-0002-6377-0833 Instituto Tocantinense Antônio Carlos SA, Brasil E-mail: victgoliveira23@gmail.com

Marcelo Henrique Amaral ORCID: https://orcid.org/0000-0002-7662-3058 Instituto Tocantinense Antônio Carlos SA, Brasil E-mail: marcelo.mha100@gmail.com

Clarisse Parrião Azevedo Cavalcante ORCID: https://orcid.org/0000-0002-4304-8018 Instituto Tocantinense Antônio Carlos SA, Brasil E-mail: dra.clariceparriao@gmail.com

Raquel Prudente de Carvalho Baldaçara ORCID: https://orcid.org/0000-0002-7817-6319 Instituto Tocantinense Antônio Carlos SA, Brasil Universidade Federal do Tocantins, Brasil E-mail: raquel.baldacara@gmail.com

\begin{abstract}
Resumo
A anafilaxia perioperatória é uma reação de hipersensibilidade imprevisível, severa e sistêmica mediada por imunoglobulinas do tipo E (IgE) ou não mediadas, de difícil diagnóstico que iminentemente pode ser fatal. Ainda, é importante citar que a anafilaxia mediada por IgE perioperatória tem relação direta com agentes administrados intravenosos, ocorrendo após anestesia, com agentes bloqueadores neuromusculares (BNMs) e antibióticos sendo os principais agentes causais. Após a administração do alérgeno, a evolução do paciente pode oscilar entre apenas efeitos colaterais, similares aos das medicações em uso, até evoluções graves como paradas cardiorrespiratórias, evidenciando uma necessidade de ser difundido o domínio teórico e técnico do manejo dessas situações para se obter melhores prognósticos. A base do tratamento da anafilaxia perioperatória inclui: manter a via aérea pérvia, fornecimento de oxigênio a 100\%, administração de adrenalina intramuscular (IM) ou intravenosa (IV) e reposição de volume. Sendo assim, visto que o paciente pode vir a óbito em minutos, devido ao quadro agudo se intensificar de forma rápida, faz-se necessário a urgência no tratamento. E para isso, o presente trabalho traz a elucidação de critérios diagnósticos (quadro clínico, evolução e exames associados). Além disso, esclarece o manejo para tratamento de pacientes em quadros de anafilaxia perioperatória. Para essa pesquisa bibliográfica, utilizou-se como referência as bases de dados do PubMed, Literatura Latino-americana e do Caribe em Ciências da Saúde (LILACS) e MEDLINE.
\end{abstract}

Palavras-chave: Anafilaxia perioperatória; Choque anafilático; Reação anafilática.

\begin{abstract}
Perioperative anaphylaxis is an unpredictable, severe and systemic hypersensitivity reaction mediated by IgE or nonmediated immunoglobulins, difficult to diagnose and potentially fatal. Furthermore, it is important to mention that perioperative IgE-mediated anaphylaxis is directly related to agents administered intravenously, occurring after anesthesia, with neuromuscular blocking agents (NMBs) and antibiotics being the main causal agents. After administration of the allergen, the evolution of the patient can range from only side effects, similar to those of medications in use, to serious evolutions such as cardiorespiratory arrests, showing a need to spread the theoretical and technical domain of the management of these situations to obtain better results. predictions. The mainstay of treatment for perioperative anaphylaxis includes: maintaining the airway patent, providing $100 \%$ oxygen,
\end{abstract}


administering intramuscular (IM) or intravenous (IV) epinephrine, and volume replacement. Thus, since the patient can die in minutes, due to the acute situation intensifying quickly, urgent treatment is necessary. And for that, the present work brings the elucidation of diagnostic criteria (clinical picture, evolution and associated exams). In addition, it clarifies the management for the treatment of patients in perioperative anaphylaxis. For this bibliographical research, the databases of PubMed, Latin American and Caribbean Literature in Health Sciences (LILACS) and MEDLINE were used as reference.

Keywords: Perioperative anaphylaxis; Anaphylactic shock; Anaphylactic reaction.

\begin{abstract}
Resumen
La anafilaxia perioperatoria es una reacción de hipersensibilidad sistémica, grave e impredecible mediada por inmunoglobulinas de tipo E (IgE) o no mediadas, difícil de diagnosticar y potencialmente mortal. Además, es importante mencionar que la anafilaxia perioperatoria mediada por IgE está directamente relacionada con los agentes administrados por vía intravenosa, ocurriendo después de la anestesia, siendo los agentes bloqueadores neuromusculares (BNM) y los antibióticos los principales agentes causales. Tras la administración del alérgeno, la evolución del paciente puede ir desde efectos secundarios únicamente, similares a los de los medicamentos en uso, hasta evoluciones graves como las paradas cardiorrespiratorias, mostrando la necesidad de difundir el dominio teórico y técnico del manejo de estas situaciones para obtener mejores resultados, predicciones. La base del tratamiento para la anafilaxia perioperatoria incluye: mantener la vía aérea permeable, proporcionar oxígeno al $100 \%$, administrar epinefrina intramuscular (IM) o intravenosa (IV) y reposición de volumen. Por lo tanto, dado que el paciente puede morir en minutos, debido a que la situación aguda se intensifica rápidamente, es necesario un tratamiento urgente. Y para eso, el presente trabajo trae la elucidación de criterios diagnósticos (cuadro clínico, evolución y exámenes asociados). Además, aclara el manejo para el tratamiento de pacientes en anafilaxia perioperatoria. Para esta investigación bibliográfica se utilizaron como referencia las bases de datos de PubMed, Literatura Latinoamericana y Caribeña en Ciencias de la Salud (LILACS) y MEDLINE.
\end{abstract}

Palabras clave: Anafilaxia perioperatoria; Choque anafiláctico; Reaccion anafiláctica.

\title{
1. Introdução
}

A anafilaxia perioperatória é uma reação de hipersensibilidade imprevisível, severa e sistêmica mediada por imunoglobulinas do tipo E (IgE) ou não mediadas, de difícil diagnóstico que iminentemente pode ser fatal. Além disso, o cenário perioperatório é complexo, incluindo vários medicamentos administrados simultaneamente combinados com os efeitos do manejo anestésico e cirúrgico (Garvey et al., 2019). Ainda, é importante citar que a anafilaxia mediada por IgE perioperatória tem relação direta com agentes administrados intravenosos, ocorrendo após anestesia, com agentes bloqueadores neuromusculares (BNMs) e antibióticos sendo os principais agentes causais (Dewachter \& Savic, 2019).

Em relação ao mecanismo, a anafilaxia usualmente é mediada por IgE ligada a receptores de mastócitos e basófilos localizados na membrana plasmática. A partir disso, há duas fases: a sensibilização e a reexposição ao alérgeno. Assim, na segunda fase acontece a liberação dos mediadores inflamatórios pré-formados (como a histamina e triptase) e os recémformados mediadores inflamatórios (leucotrienos, tromboxano A 2, citocinas), levando ao aparecimento de manifestações clínicas (Dewachter \& Savic, 2019).

Ademais, cabe ressaltar que a hipersensibilidade imediata perioperatória também pode ser desencadeada por outros mecanismos, tais como os não alérgicos, incluindo ativação de mastócitos e liberação de histamina induzida por agentes de bloqueio neuromuscular (Dewachter \& Savic, 2019).

Sobre a epidemiologia, de acordo com os dados coletados nas bases de publicações da França nos últimos 25 anos, essa incidência de reações mediadas por IgE são estimadas sendo 1 para 10000. Já em regiões como Noruega de 1 para 6000 , Australia 1 em 20000 (Yoon, Bang, Seo \& Hong, 2014). Além disso, publicação recente realizada no Reino Unido pela National Audit Projects (NAP6), levando em conta apenas reações anafiláticas ou casos fatais a incidência se revelou em 1 para 10000. Contudo, há subnotificação dos casos por dados incompletos no preenchimento das informações, de acordo com os autores. (Garvey et al., 2019). Pontua-se ainda, que essa variação é multifatorial, incluindo desde diferenças nas práticas locais, preferência de medicamentos, práticas de encaminhamento, até fatores de genéticos e ambientais (Garvey et al., 2019).

Assim, distribui-se os casos anafiláticos assumindo as respectivas porcentagens de incidência: bloqueadores 
neuromusculares como agentes em 50 a 70\%, látex em 12\% a 16\%, antibióticos ficando com 15\%, hipnóticos com 3,0\% e opioides com 1,3\% (Haque \& Nossaman, 2012). Já em questão de prevalência o quadro de anafilaxia tem maior reiteração no sexo feminino aproximadamente 3:1 se comparado com o sexo masculino, tendo uma ligação direta com os hormônios sexuais (Lavery \& Bernstein, 2019).

Após a administração do alérgeno, a evolução do paciente pode oscilar entre apenas efeitos colaterais, similares aos das medicações em uso, até evoluções graves como paradas cardiorrespiratórias, evidenciando uma necessidade de ser difundido o domínio teórico e técnico do manejo dessas situações para se obter melhores prognósticos. Além disso, visto que o diagnóstico de uma crise anafilática seja essencialmente clínico, é considerável que os casos de anafilaxia perioperatória estejam de certa forma epidemiológica sendo banalizados, causando certa discrepância documental/real (Nel \& Eren, 2011).

Portanto, sabendo que as formas de diagnósticos para os quadros de anafilaxia são clínicos, é relevante pontuar que a evolução do quadro do paciente é agudo e característico, sendo os sinais sistêmicos classificados da seguinte forma: classe I sinais cutâneos generalizados (eritema, urticária com ou sem angioedema), classe II - envolvimento moderado de vários órgãos com sinais cutâneos, hipotensão e taquicardia, hiper-reatividade brônquica, classe III - envolvimento sistêmico de órgãos, risco de vida grave que necessita de tratamento específico (colapso, taquicardia ou bradicardia, arritmias cardíacos, broncoespasmo) e classe IV - parada circulatória/respiratória (Pajno et al., 2011).

Nesse contexto, como terapia inicial preconiza-se o cessamento do agente causador, manter vias aéreas, ofertar O2 a $100 \%$ e reanimação volêmica, importante para reversão do quadro anormal da volemia, que surge pela soma do aumento do calibre dos vasos advinda da histamina. Além disso, faz-se necessário a administração da adrenalina por sua ação a-agonista, que diminui o calibre dos vasos e por sua ação beta-adrenérgica, que causa broncodilatação, aumento do ritmo cardíaco e diminuição da resposta inflamatória por supressão da liberação dos compostos pró-inflamatórios (Spindola et al., 2020).

Já na terapia secundária, recomenda-se o uso de corticosteroides e anti-histamínicos que também são destinados para os casos de classe I. Dessa forma, ambos os medicamentos são válidos como tratamento para reações sistêmicas e anafilaxia, pois ajudam a prevenir edema, sintomas cutâneos e uma recaída de as reações sistêmicas que podem ocorrer até 24 h após a reação inicial (Pajno et al., 2011).

Sendo assim, visto que o paciente pode vir a óbito em minutos, devido ao quadro agudo se intensificar de forma rápida, faz-se necessário a urgência no tratamento. E para isso, o presente trabalho traz a elucidação de critérios diagnósticos (quadro clínico, evolução e exames associados). Além disso, esclarece o manejo para tratamento de pacientes em quadros de anafilaxia perioperatória.

\section{Metodologia}

Trata-se de uma revisão narrativa de literatura seguindo os critérios de suporte metodológico da Dra. Edna Terezinha Rother. Foram selecionados os principais artigos e diretrizes sobre anafilaxia perioperatória, incluindo os critérios clínicos para diagnóstico e manejo do paciente. Para essa pesquisa bibliográfica, utilizou-se como referência as bases de dados do PubMed, Literatura Latino-americana e do Caribe em Ciências da Saúde (LILACS) e MEDLINE. Assim, os descritores aplicados foram: anafilaxia perioperatória, choque anafilático e reação anafilática, e os artigos passaram por um processo de seleção e revisão.

Os dados para utilização foram referenciados dos últimos 10 anos, ou seja, entre 2011 e 2021. Sobre o idioma, a pesquisa não se restringiu ao português, mas também, inglês e espanhol. Além disso, o filtro para tornar os artigos utilizáveis incluía ter o conteúdo totalmente disponível.

Em relação a confecção do artigo, deu-se de acordo com os seguintes passos: elaboração do tema pertinente com material compactuado no intuito da revisão, definição dos descritores a serem utilizados, análise dos limiares de utilização ou não dos documentos, seleção do acervo documental e retirada de artigos não pertinentes e por fim, a elaboração do artigo. 


\section{Resultados}

Os dados foram coletados nas bases de dados do Pubmed, Medline e Lilacs, que são plataformas de livre acesso. Os critérios de inclusão utilizados foram: anafilaxia perioperatória, choque anafilático e reação anafilática, sendo encontrados um total de 366 artigos. Ao final da seleção, por meio dos seguintes filtros: com o conteúdo totalmente disponível, redigidos em português, inglês, espanhol, durante o período dos últimos 10 anos de publicação, restaram um total de 125 artigos. Além disso, foram retirados os artigos repetidos.

\section{Discussão}

\section{Epidemiologia e principais causas de anafilaxia}

A anafilaxia como tipo de doença foi descrita há mais de 100 anos por Charles Richet e por Paul Portier, que pela busca de um antídoto acabaram por provocar uma reação anafilática em cães de laboratório (Moura et al., 2020). Por esse estudo ficou evidenciado que a anafilaxia tem como definição uma reação sistêmica grave, que pode ocorrer de forma aguda através de vias como: por ingestão, inalação, contato e administração medicamentosa (Ribeiro, Chong Neto \& Rosario Filho, 2017).

A reação anafilática é a representação mais severa de uma reação de hipersensibilidade que pode ser subdivididas em alérgicas (mediadas por Imunoglobulina tipo E) ou não mediadas (excluindo o mecanismo imunológico) (Caimmi et al., 2011). Essa reação se dá na maioria das vezes de forma imunomediada por IgE, ou, de forma menos frequente não mediada (Manfredi et al. 2013).

Em prevalência de casos de anafilaxia perioperatória essas reações são parcialmente raras, visto que, sua ocorrência abrangem 1 em 10000-20000 casos de aplicações em cenários de anestesias por bloqueadores neuromusculares (BNM) (Lagopoulos \& Gigi 2011).

Diante desse cenário, um estudo francês analisou os dados de oito anos, dos períodos de 1997 a 2004 com os principais causadores de anafilaxia nas práticas de anestesiologia. Esse resultado apresentou como causa mais comum os BNM que são responsáveis por $69.2 \%$ das reações alérgicas, seguidos pelo látex com $12.1 \%$ e os antibióticos com $8 \%$ dos valores totais, esses tipos de reações de hipersensibilidade mediada por IgE (Misbah \& Krishna, 2019). Com prevalência de incidência anual no gênero feminino (Lavery \& Bernstein, 2019).

\section{Fisiopatologia}

A manifestação clínica da anafilaxia vai derivar de acordo com a liberação aguda de mediadores causados pela degranulação de mastócitos ou em menor número de basófilos (que estão implicados nas reações IgE dependente e IgE independentes) (Turkalj et al., 2019). Essa apresentação clínica do paciente inicia-se de diversas vezes por comprometimento cutâneo (urticária e angioedema) e com possíveis agravamentos dos aparelhos acometidos como, respiratório (broncoespasmo e obstrução das vias aéreas superiores), cardiovascular (hipotensão e arritmias), sistema nervoso e trato gastrointestinal (cólicas abdominais, náuseas e vômito) (Moura et al., 2020).

Assim, tais reações do organismo decorrem de um processo de exposição inicial do paciente ao alérgeno que leva a uma maciça liberação de mediadores teciduais (mastócitos ou basófilos). Essa exposição predispõe o organismo a produção e liberação de $\mathrm{IgE}$, que se ligam a receptores de alta afinidade, que provocando o início da cascata com liberação de histamina, proteases, proteoglicanos e fatores ativadores de plaquetas (Lagopoulos \& Gigi 2011).

De forma específica, a histamina provoca um aumento da permeabilidade vascular e uma vasodilatação associada a prostaglandina que afeta os sistemas respiratórios causando broncoconstrição pulmonar (Nel \& Eren, 2011). Dessa forma, essa 
liberação provoca uma reação sistêmica severa, que acarreta uma ausência de um consenso em determinar seu diagnóstico de forma assertiva (Ribeiro, Chong Neto \& Rosario Filho, 2017).

\section{Diagnóstico e diagnóstico diferencial}

O quadro de reação anafilática deve ser diagnosticado de forma precoce, dado que, o curso da doença propicia a uma evolução rápida e possível óbito do paciente. Desta forma, é importante a utilização de critérios diagnósticos, como a triptase sérica, facilitando para profissionais da saúde o manejo desses pacientes, visto que, os parâmetros totais provêm valores de sensibilidade e especificidade de até 95\% (Ribeiro, Chong Neto \& Rosario Filho, 2017).

A triptase é uma serina-protease de grânulos secretores, abundante nos mastócitos, podendo assim, ser utilizada como biomarcador. Seu principal objetivo diagnóstico é sua quantificação no momento da reação, confirmando a degranulação dos mastócitos (Garvey et al., 2019). Sua concentração total é medida no plasma ou soro, $\left(\mathrm{N}<11-13 \mu \mathrm{g} \mathrm{L}^{-1}\right.$ ), atingindo pico entre 15 minutos e 2 horas após o início da anafilaxia (Dewachter \& Savic, 2019). Assim, confirma-se sua utilidade como um dos meios diagnósticos complementares, já que os níveis séricos fazem relação direta com a gravidade do quadro do paciente.

Os sintomas mais comuns se apresentam de forma cutânea (urticária e angioedema) em torno de 90\%, respiratórios (dispneia e sibilância) de 55 a $60 \%$ dos casos e podem estar acompanhados de sintomas menos frequentes como tontura, síncope, hipotensão, visão turva, náuseas, vômitos, diarreia, cefaleia e possíveis convulsões (Marinho et al. 2016). Embora as manifestações cutâneas estejam presentes na maioria das crises, essas podem não se apresentarem em casos que se associem a choque (Yoon, Bang, Seo \& Hong, 2014).

A gravidade do quadro do paciente está diretamente relacionada com o agente causal e a forma de contato. Diante disso, a média das manifestações associadas com o sistema cardíaco e respiratório foram de aproximadamente 30 minutos em alimentos e de cinco minutos em medicações ou contrastes utilizados em procedimentos perioperatório (Misbah \& Krishna, 2019). Assim, é de suma importância para os critérios de diagnóstico a facilidade e eficácia dos métodos a serem seguidos, uma vez que, existem quadros clínicos que são facilmente confundidos com as reações alérgicas, como a reação vasomotora (nervo vasovagal).

Essa reação inicia-se com congestão venosa propiciando a redução do retorno venoso e subsequente ativação dos receptores mecanossensoriais, sentidos que ativam o nervo vago e causam redução da frequência cardíaca, vasodilatação excessiva e possível perda da consciência. A síncope vasovagal é mediada por reflexos que incluem pródromo de tonturas, com ou sem náuseas, palidez e/ou sudorese (Au, Lau, Lam \& Chan, 2020). Ainda, há outras causas com menores semelhanças, como os quadros de bradicardias provocados por betabloqueador, que apesar de menos frequentes ainda necessitam de um diagnóstico diferencial assertivo.

A anafilaxia é a origem de uma reação adversa imprevisível e potencialmente letal, portanto, com objetivo de reduzir o risco no processo perioperatório deve-se preveni-la. Assim, para pacientes com histórico prévio de reações alérgicas ou que em algum momento apresentam reação ao látex ou produtos que possam ser utilizados durante os procedimentos, se faz necessário a anamnese com detalhamento dos quadros reacionais e que esse processo deve ser avaliado por um anestesista ou alergista que pode pedir teste de sensibilização para sua identificação (Caffarelli et al., 2011).

\section{Manejo da anafilaxia}

A base do tratamento da anafilaxia perioperatória inclui: manter a via aérea pérvia, fornecimento de oxigênio a 100\%, administração de adrenalina intramuscular (IM) ou intravenosa (IV) e reposição de volume. Importante salientar que a adrenalina é efetiva quando há o reconhecimento precoce da anafilaxia e forem administradas rapidamente doses adequadas (Yim, 2016). Além disso, o meio de administração do medicamento é preferencialmente via IM na porção lateral da coxa, 
decurso da sua facilidade e agilidade de administração, enquanto a via IV deve ser feita por especialista como anestesista ou intensivista que tem proficiência no seu uso e requer a monitorização contínua pois a administração em dosagens superiores ou inferiores à adequadas podem causar severas consequências (Spindola et al., 2020).

Diante dos critérios de gravidade da reação anafilática, são preconizados os seguintes tratamentos: na classe I são feitas apenas o acompanhamento da progressão do paciente e avaliação de possível edema de laringe, classe II já se faz a necessidade da intervenção direta, com a aplicação de adrenalina IM 200-300 $\mu \mathrm{g}$ ou podendo ser usada IV 10-20 $\mu \mathrm{g}$ (bolus) se não houver melhora do quadro, repetindo em 2 minutos, classe III aumenta-se a dosagem de adrenalina administrada, tanto na

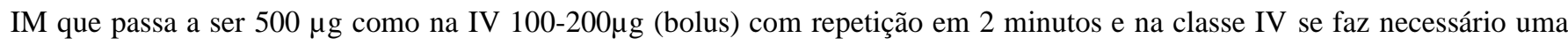
administração máxima da dose seguindo o protocolo de RCP, 1mg (bolus). Por fim, a partir da classe II há administração do volume de $20 \mathrm{~mL} / \mathrm{Kg}$ em bolus, com repetição de cristaloide, não coloide (Spindola et al., 2020).

Além disso, é importante ressaltar que o posicionamento do paciente é importante para melhorar o retorno venoso e em casos que não obtiver melhora no tratamento de primeira linha, pode-se fazer a administração de outros vasopressores como: noradrenalina ou metaraminol. Ainda, em casos de broncoespasmos resistentes pode ser tratado com o uso de salbutamol IV (Yim, 2016).

Em casos específicos como nas anafilaxias refratárias, leva-se em conta novamente o questionamento acerca da remoção dos agentes causadores, a coleta de triptase sérica em duas amostras, sendo a primeira em até uma hora do início do quadro e a segunda de duas a quatro horas. Associado a isso, se o paciente mantiver o quadro de hipotensão após 10 minutos sem resposta da adrenalina, direciona-se ao uso de noradrenalina, vasopressina e glucagon (em caso de pacientes que fazem o uso de betabloqueador) (Spindola et al., 2020).

Outrossim, em relação as particularidades como na gestante é importante ressaltar condutas como deslocamento do útero para a esquerda, com intuito de descomprimir a aorto-cava que deverá ser mantida durante todo o quadro de ressuscitação. Ainda, deve-se considerar a cesariana de emergência, com objetivo de facilitar a ressuscitação da mãe (Kolawole, Marshall, Crilly, Kerridge \& Roessler, 2017).

\section{Conclusão}

Devido ao potencial de letalidade das reações de anafilaxia perioperatória durante os procedimentos, evidenciou-se a utilidade de um manejo organizado e rápido, relacionado a uma prevenção por averiguação de intolerâncias ou alergias em pacientes de forma prévia. Com isso, ter uma análise sistemática do histórico médico do paciente, permitindo ao procedimento uma maior segurança durante o período perioperatório.

Diante disso, explicitou-se a necessidade de uma identificação criteriosa acerca dos tipos de agentes causadores, para que seja possível sua investigação e remoção rápida em casos de reação anafilática. Nesse caso, conseguiu-se levantar que entre as principais causas se encontram os bloqueadores neuromusculares, seguidos do látex e antibióticos.

Associado a esse manejo, evidencia-se a importância de sistematizar o diagnóstico do paciente, visto que, o único exame de auxílio à clínica será o pedido da dosagem de triptase sérica para a confirmação do quadro anafilático. Outrem, esse estudo se faz importante para alertar aos clínicos gerais e especialistas que a reação anafilática pode ocorrer em qualquer momento perioperatório e a intervenção deve ser imediata, em conjunto a protocolos de manejo que permitirá o tratamento adequado e confirmação do quadro.

Dessa forma, sugerimos que esse trabalho seja o apoio inicial em futuras pesquisas que procurem pelo tema de anafilaxia perioperatória, visto a necessidade de seguir um protocolo no atendimento, buscando sempre uma maior rapidez e eficácia ao tratamento do paciente. Assim, se faz necessário a atualização na eventualidade de novas descobertas de causas, critérios diagnósticos ou mudanças na abordagem dos quadros anafiláticos. 


\section{Agradecimentos}

Agradecemos à Prof. Dra. Lorena Dias Monteiro pela oportunidade de realizar este artigo durante a disciplina de TCC do curso de Medicina ITPAC-Palmas.

\section{Referências}

Au, E., Lau, C. S., Lam, K., \& Chan, E. (2020). Perioperative anaphylaxis and investigations: a local study in Hong Kong. Singapore medical journal, 61(4), 200-205. https://doi.org/10.11622/smedj.2019156

Caffarelli, C., Stringari, G., Miraglia Del Giudice, M., Crisafulli, G., Cardinale, F., Peroni, D. G., \& Bernardini, R. (2011). Prevention of allergic reactions in anesthetized patients. International journal of immunopathology and pharmacology, 24(3 Suppl), S91-S99. https://doi.org/10.1177/03946320110240s313

Caimmi, S., Caimmi, D., Bernardini, R., Caffarelli, C., Crisafulli, G., Pingitore, G., \& Marseglia, G. L. (2011). Perioperative anaphylaxis: epidemiology. International journal of immunopathology and pharmacology, 24(3 Suppl), S21-S26. https://doi.org/10.1177/03946320110240s304

Dewachter, P., \& Savic, L. (2019). Perioperative anaphylaxis: pathophysiology, clinical presentation and management. BJA education, 19(10), 313-320. https://doi.org/10.1016/j.bjae.2019.06.002

Garvey, L. H., Dewachter, P., Hepner, D. L., Mertes, P. M., Voltolini, S., Clarke, R., Cooke, P., Garcez, T., Guttormsen, A. B., Ebo, D. G., Hopkins, P. M., Khan, D. A., Kopac, P., Krøigaard, M., Laguna, J. J., Marshall, S., Platt, P., Rose, M., Sabato, V., Sadleir, P., \& Kolawole, H. (2019). Management of suspected immediate perioperative allergic reactions: an international overview and consensus recommendations. British journal of anaesthesia, 123(1), e50e64. https://doi.org/10.1016/j.bja.2019.04.044

Haque, S. H., \& Nossaman, B. D. (2012). Dyed but not dead. The Ochsner journal, 12(2), 135-140.

Kolawole, H., Marshall, S. D., Crilly, H., Kerridge, R., \& Roessler, P. (2017). Australian and New Zealand Anaesthetic Allergy Group/Australian and New Zealand College of Anaesthetists Perioperative Anaphylaxis Management Guidelines. Anaesthesia and intensive care, 45(2), 151-158. https://doi.org/10.1177/0310057X1704500204

Lagopoulos, V., \& Gigi, E. (2011). Anaphylactic and anaphylactoid reactions during the perioperative period. Hippokratia, 15(2), 138-140.

Lavery, W. J., \& Bernstein, J. A. (2019). Cyclical hypersensitivity, anaphylaxis, and related hormonal reaction. Annals of allergy, asthma \& immunology: official publication of the American College of Allergy, Asthma, \& Immunology, 122(2), 140-147. https://doi.org/10.1016/j.anai.2018.11.016

Manfredi, G., Pezzuto, F., Balestrieri, A., Lo Schiavo, M., Montera, M. C., Pio, A., Iannelli, M., Gargano, D., Bianchi, M. J., Casale, G., Galimberti, M., Triggiani, M., \& Piazza, O. (2013). Perioperative anaphylactic risk score for risk-oriented premedication. Translational medicine @ UniSa, 7, $12-17$.

Marinho, S., Kemp, H., Cook, T. M., Farmer, L., Farooque, S., Lucas, D. N., Garcez, T., Floss, K., Torevell, H., Thomas, M., Warner, A., Hitchman, J., Ferguson, K., Egner, W., Nasser, S., Karanam, S., Kong, K. L., McGuire, N., Bellamy, M., \& Harper, N. (2018). Cross-sectional study of perioperative drug and allergen exposure in UK practice in 2016: the 6th National Audit Project (NAP6) Allergen Survey. British journal of anaesthesia, 121(1), 146-158. https://doi.org/10.1016/j.bja.2018.04.016

Misbah, S. A., \& Krishna, M. T. (2019). Peri-Operative Anaphylaxis-An Investigational Challenge. Frontiers in immunology, 10, 1117. https://doi.org/10.3389/fimmu.2019.01117

Moura, A. L., Regateiro, F., Coutinho, I. A., Mota, D. S., Paiva, A., Bom, A. T., \& Faria, E. (2020). Recurrent peripartum anaphylaxis as a manifestation of systemic mastocytosis. Revista portuguesa de imunoalergologia, 28(4), 241-246. https://doi.org/http://doi.org/10.32932/rpia.2020.12.047

Nel, L., \& Eren, E. (2011). Peri-operative anaphylaxis. British journal of clinical pharmacology, 71(5), 647-658. https://doi.org/10.1111/j.13652125.2011.03913.x

Pajno, G. B., Crisafulli, G., Caminiti, L., Marseglia, G. L., Cardinale, F., Paravati, F., \& Caffarelli, C. (2011). Perioperative allergy: therapy. International journal of immunopathology and pharmacology, 24(3 Suppl), S101-S104. https://doi.org/10.1177/03946320110240s314

Ribeiro, M., Chong Neto, H. J., \& Rosario Filho, N. A. (2017). Diagnosis and treatment of anaphylaxis: there is an urgent needs to implement the use of guidelines. Einstein (Sao Paulo, Brazil), 15(4), 500-506. https://doi.org/10.1590/S1679-45082017RW4089

Spindola, M., Solé, D., Aun, M. V., Azi, L., Bernd, L., Garcia, D. B., Capelo, A. V., Cumino, D. O., Lacerda, A. E., Lima, L. C., Morato, E. F., Nunes, R. R., Rubini, N., da Silva, J., Tardelli, M. Â., Watanabe, A. S., Curi, E. F., \& Sano, F. (2020). Atualização sobre reações de hipersensibilidade perioperatória: documento conjunto da Sociedade Brasileira de Anestesiologia (SBA) e Associação Brasileira de Alergia e Imunologia (ASBAI) - Parte I: tratamento e orientação pós-crise [Update on perioperative hypersensitivity reactions: joint document of the Brazilian Society of Anesthesiology (SBA) and Brazilian Association of Allergy and Immunology (ASBAI) - Part I: post-crisis guidelines and treatment]. Brazilian journal of anesthesiology (Elsevier), 70(5), 534548. https://doi.org/10.1016/j.bjan.2020.06.004

Rother, E. T., \& Braga, M. E. R. (2005). Como Elaborar Sua Tese: Estrutura e Referências (4th ed.). BC Gráfica.

Spindola, M., Solé, D., Aun, M. V., Azi, L., Bernd, L., Garcia, D. B., Capelo, A. V., Cumino, D. O., Lacerda, A. E., Lima, L. C., Morato, E. F., Nunes, R. R., Rubini, N., da Silva, J., Tardelli, M. Â., Watanabe, A. S., Curi, E. F., \& Sano, F. (2020). Atualização sobre reações de hipersensibilidade perioperatória: documento conjunto da Sociedade Brasileira de Anestesiologia (SBA) e Associação Brasileira de Alergia e Imunologia (ASBAI) - Parte I: tratamento e orientação pós-crise [Update on perioperative hypersensitivity reactions: joint document of the Brazilian Society of Anesthesiology (SBA) and Brazilian Association of Allergy and Immunology (ASBAI) - Part I: post-crisis guidelines and treatment]. Brazilian journal of anesthesiology (Elsevier), 70(5), 534 548. https://doi.org/10.1016/j.bjan.2020.06.004 
Research, Society and Development, v. 10, n. 13, e375101321423, 2021

(CC BY 4.0) | ISSN 2525-3409 | DOI: http://dx.doi.org/10.33448/rsd-v10i13.21423

Solé, D., Spindola, M., Aun, M. V., Araújo Azi, L., Bernd, L., Garcia, D. B., Capelo, A. V., Cumino, D. O., Lacerda, A. E., Lima, L. C., Morato, E. F., Nunes, R. R., Rubini, N., da Silva, J., Tardelli, M. A., Watanabe, A. S., Curi, E. F., \& Sano, F. (2020). Atualização sobre reações de hipersensibilidade perioperatória: documento conjunto da Sociedade Brasileira de Anestesiologia (SBA) e Associação Brasileira de Alergia e Imunologia (ASBAI) - Parte II: etiologia e diagnóstico [Update on perioperative hypersensitivity reactions: joint document from the Brazilian Society of Anesthesiology (SBA) and Brazilian Association of Allergy and Immunology (ASBAI) - Part II: etiology and diagnosis]. Brazilian journal of anesthesiology (Elsevier), 70(6), 642-661. https://doi.org/10.1016/j.bjan.2020.08.008

Turkalj, M., Erceg, D., Martinuš, M., Babić, E., Karin, M., \& Bevanda, M. (2019). Diagnosis of perioperative anaphylaxis. Psychiatria Danubina, 31(Suppl 1), 50-59.

Yim Rebecca. (2016). An Update on Perioperative anaphylaxis. World Federations Societies of Anesthesiologists. ATOTW Tutorial 324. págs. 1-6.

Yoon, S. H., Bang, J. Y., Seo, H., \& Song, J. G. (2014). Sudden cardiovascular collapse caused by severe anaphylaxis after cisatracurium use: a case report. Korean journal of anesthesiology, 67(6), 412-415. https://doi.org/10.4097/kjae.2014.67.6.412 